\title{
Necessity of Flanking Repeats R1' and R8' of Human Pumilio1 Protein for RNA Binding
}

Kento Nakamura, Taishu Nakao, Tomoaki Mori, Serika Ohno, Yusuke Fujita, Keisuke

Masaoka, Kazuki Sakabayashi, Koichi Mori, Takamasa Tobimatsu, and Takashi Sera*

Department of Applied Chemistry and Biotechnology, Graduate School of Interdisciplinary Science and Engineering in Health Systems, Okayama University, Tsushima-Naka, Kita-ku, Okayama 700-8530, Japan

*Corresponding author:

Takashi Sera

E-mail: sera@cc.okayama-u.ac.jp

\section{Supporting Information}

Contents

1. Figure $\mathrm{S}$

P. S2

2. Figure $\mathrm{S} 2$

P. S3

3. Figure $\mathrm{S} 3$

P. S4 

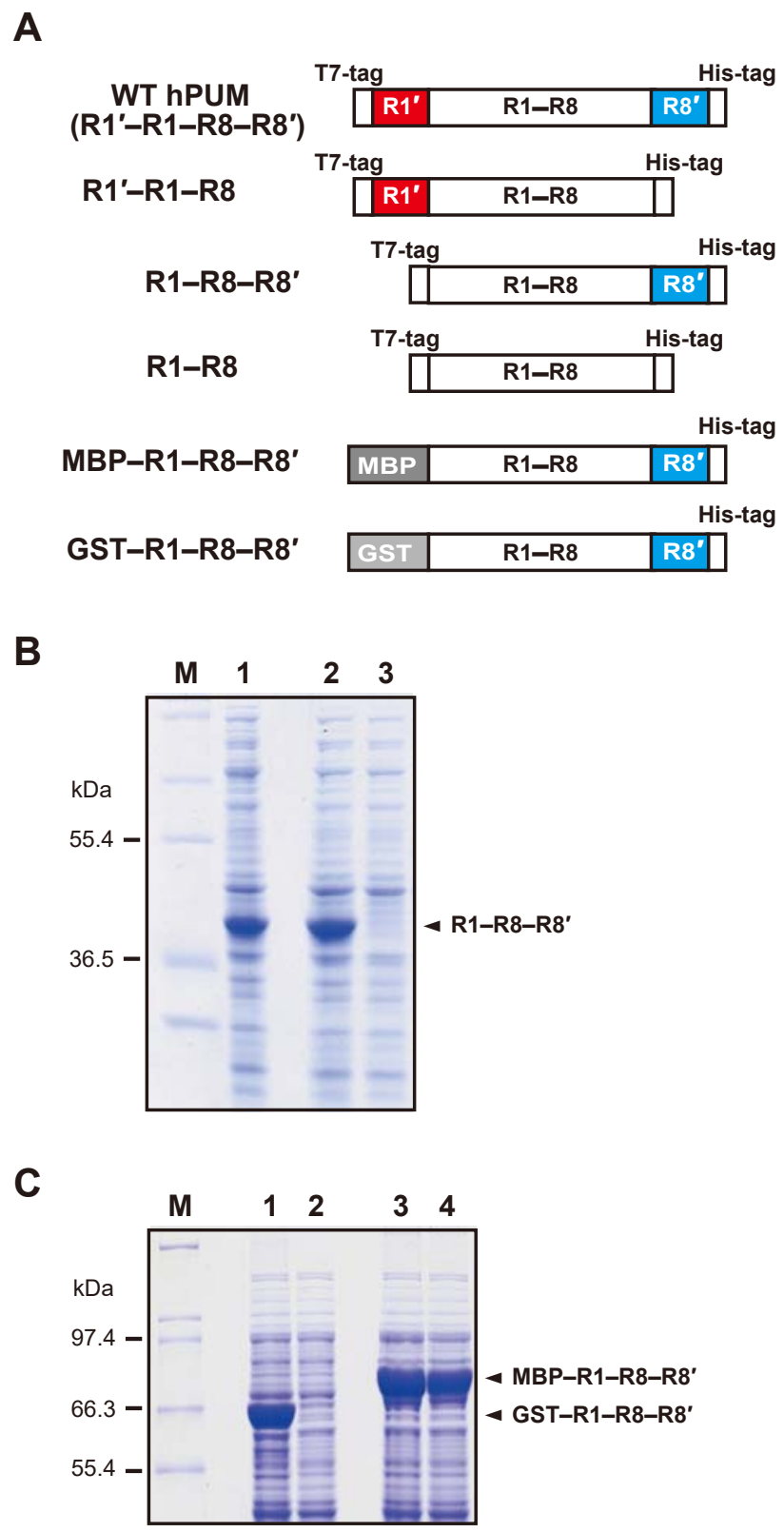

Figure S1. Structures of hPUM and its generated deletion mutants and improvement of solubility of hPUM deletion mutants by fusing to MBP. (A) Schematic representation of WT hPUM and three deletion mutants, R1'-R1-R8, R1-R8-R8', and R1-R8. (B) sodium dodecyl sulfatepolyacrylamide gel electrophoresis (SDS-PAGE) analysis of the R1-R8-R8' deletion mutant after sonication of E. coli expressing the mutant. Lane M: a protein size marker (Mark12 Wide Range Unstained Standard, Invitrogen), lane 1: E. coli expressing R1-R8-R8', lane 2: suspension after sonication of E. coli (shown in lane 1), lane 3: supernatant after centrifugation of the suspension (shown in lane 2). (C) Comparison of solubilization efficiencies of GST and MBP fusion for the R1-R8-R8' deletion mutant. Lane M: a protein size marker (Mark12 Wide Range Unstained Standard, Invitrogen), lane 1: suspension after sonication of E. coli expressing GST-R1-R8-R8' fusion, lane 2: supernatant after centrifugation of the suspension (shown in lane 1), lane 3: suspension after sonication of E. coli expressing MBP-R1-R8-R8' fusion, lane 4: supernatant after centrifugation of the suspension (shown in lane 3 ). 


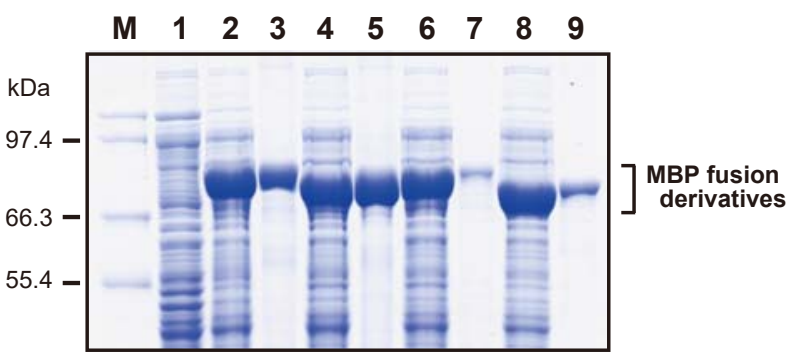

Figure S2. Preparation of MBP-fusion derivatives of WT hPUM and three deletion mutants shown in Figure 1B. SDS-PAGE analysis of recombinant E. coli after induction of expression of WT hPUM and three deletion mutants, respectively, and purified proteins of WT hPUM and three deletion mutants. Lane M: a protein size marker (Mark12 Wide Range Unstained Standard, Invitrogen), lane 1: non-recombinant E. coli BL21(DE3), lane 2: recombinant E. coli after induction of MBP-WT hPUM expression, lane 3: purified MBP-WT hPUM protein, lane 4: recombinant E. coli after induction of MBP-R1'-R1-R8 expression, lane 5: purified MBP-R1'-R1-R8 protein, lane 6: recombinant E. coli after induction of MBP-R1-R8-R8' expression, lane 7: purified MBP-R1-R8-R8' protein, lane 8: recombinant E. coli after induction of MBP-R1-R8 expression, lane 9: purified MBP-R1-R8 protein. 


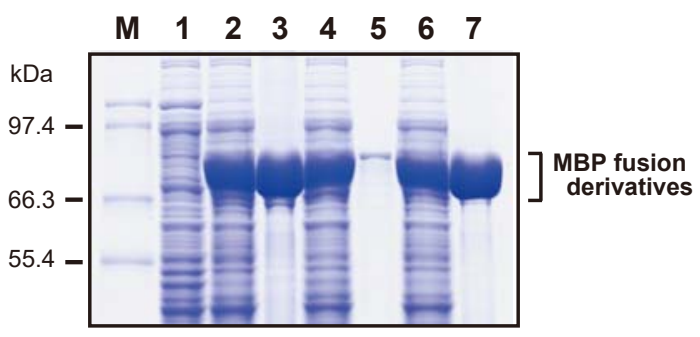

Figure S3. Preparation of MBP-fusion derivatives of hPUM alanine mutants shown in Figure 3A. SDS-PAGE analysis of recombinant E. coli after induction of expression of these alanine mutants, respectively, and purified proteins of WT hPUM and these alanine mutants. Lane M: a protein size marker (Mark12 Wide Range Unstained Standard, Invitrogen), lane 1: non-recombinant E. coli BL21(DE3), lane 2: recombinant E. coli after induction of MBP-hPUM_R8' (Ala) expression, lane 3: purified MBP-hPUM_R8' (Ala) protein, lane 4: recombinant E. coli after induction of MBP-hPUM_R1' (Ala) expression, lane 5: purified MBP-hPUM_R1' (Ala) protein, lane 6: recombinant E. coli after induction of MBP-hPUM_R8' His (Ala) expression, lane 7: purified MBP-hPUM_R8' His (Ala) protein. 